Dochead : Hémodialyse

\title{
Prescription de la dose de dialyse
}

\section{Prescribing the dose of dialysis}

Mélanie Hanoy *, Frank Le Roy, Dominique Guerrot

Service de néphrologie hémodialyse, centre hospitalier universitaire de Rouen, 147 avenue du Maréchal-Juin, 76230 Rouen cedex. France

*Auteur correspondant ; e-mail : melanie.hanoy@chu-rouen.fr ; tél. : 0232889002 


\section{Histoire de la dose de dialyse}

En 1960, Clyde Shields, à 39 ans, fut le premier patient à bénéficier de l'hémodialyse chronique intermittente pour la prise en charge d'une insuffisance rénale terminale secondaire à une glomérulopathie. Les critères permettant de juger de l'efficacité de l'épuration étaient essentiellement cliniques : reprise de l'appétit avec stabilisation du poids, contrôle de la pression artérielle et des signes de surcharge hydrosodée, absence de signes d'encéphalopathie urémique. Le rythme des séances d'épuration était défini par la réapparition des signes cliniques : asthénie, céphalées, vomissements, dyspnée (1).

Une décennie plus tard, le concept de dialyse adéquate ou efficace est né: il semblait nécessaire de déterminer pour chaque patient une durée optimale de dialyse hebdomadaire permettant au patient une stabilité clinique avec les meilleures chances de survie (2). La neuropathie périphérique était le symptôme urémique le plus difficile à contrôler justifiant 20 à 40 heures de dialyse par semaine avec les dialyseurs standards utilisés selon que le patient ait ou non une fonction rénale résiduelle. A partir d'observations cliniques, notamment en dialyse péritonéale, le groupe de Seattle dirigé par les Dr Scribner et Babb a réussi à concevoir l'idée de la responsabilité des moyennes molécules dans ces complications neurologiques et à soulever la façon de les épurer : nécessité de membranes de grande porosité et de grande surface ce qui a eu l'avantage de permettre la réduction des durées de séance : il s'agissait de l'hypothèse « square meter-hour hypothesis »(3,4). Pour des durées de dialyse hebdomadaire similaires, l'état clinique des patients dialysés trois fois par semaine était bien meilleur que celui des patients traités seulement deux fois par semaine (5).

La notion de « dose de dialyse » est apparue quelques années plus tard. Au cours de l'essai randomisé de la National Cooperative Dialysis Study (NCDS), il a été montré que la morbimortalité des patients était conditionnée par la dose de dialyse prescrite à chaque séance (6).

Après les travaux de Gotch et Sargent en 1985, l'indice Kt/V de l'urée a été considéré comme marqueur de quantification le plus pertinent cliniquement. Il se définit comme le produit de clairance effective de l'urée $(K)$ et du temps de dialyse $(t)$ rapporté au volume de diffusion corporel de l'urée $(V)$, assimilé à l'eau totale (7).

Des études observationnelles ont permis de fixer un Kt/V urée simple pool (Kt/Vsp) (compartiment plasmatique considéré) minimal de 1,2, en dessous duquel les résultats cliniques étaient défavorables, ce qui correspondait à une réduction plasmatique de l'urée de $65 \%$ au cours d'une séance de dialyse (8). L'étude prospective HEMO, multicentrique et randomisée, a validé le

concept de Kt/V urée équilibré (equilibrated $\mathrm{Kt} / \mathrm{V}=$ single pool Kt/V $-0,4 \times 60 \times \frac{K}{V}$ ) (9). En effet, la séance de dialyse déséquilibre la concentration de l'urée qui baisse davantage dans le compartiment plasmatique que dans les compartiments périphériques (peau, muscle, intracellulaire). Le Kt/V simple 
pool tend à surestimer la quantité d'urée extraite. Cette étude a comparé le pronostic des patients recevant une dose de dialyse standard (Kt/V équilibré cible : 1,05) à ceux recevant une dose de dialyse élevée (Kt/V équilibré cible : 1,45) et a montré l'absence de bénéfice significatif de doses élevées sur la morbimortalité des patients (10).

La dose de dialyse moyenne des 426 patients inclus dans le groupe « dose de dialyse standard » était de 1,32 pour le Kt/Vsp, soit un Kt/V équilibré de 1,16 et un pourcentage de réduction moyen de l'urée de 67\%. Quelques années plus tard, l'étude observationnelle, multicentrique Dialysis Outcomes and Practice Patterns Study (DOPPS) a montré des résultats équivalents, à savoir un bénéfice en terme de survie de l'augmentation des doses de dialyse jusqu'à un seuil de Kt/V équilibré d'1,4 (11). Cependant, il semble qu'il y ait un avantage significatif de Kt/V plus élevé pour les femmes et non pour les hommes.

À la fin des années 1990, trois études observationnelles tendaient à apporter une explication à ces objectifs discordants entre les sexes (12-14). Elles ont soulevé le manque de pertinence de l'utilisation du volume de distribution de l'urée pour l'évaluation pronostique des patients, notamment en termes de mortalité. En effet, une mortalité significativement plus élevée a été constatée pour des $\mathrm{Kt} / \mathrm{V}$ bas, témoignant d'une dose de dialyse insuffisante, mais également pour des $\mathrm{Kt} / \mathrm{V}$ élevés : ce résultat s'est avéré non pas en lien avec une « surdialyse », mais avec un volume de distribution de l'urée bas, témoignant d'une faible masse musculaire et donc d'un statut nutritionnel précaire avec possiblement une dose de dialyse $(K t)$ insuffisante.

Au cours d'une étude rétrospective ayant porté sur 17141 patients, Lowrie et al. ont identifié une corrélation linéaire entre l'indice $K t$ (clairance urée $\times$ durée de dialyse) et la mortalité. Ils ont noté une interaction statistique avec le sexe uniquement, les hommes requérant une dose de dialyse plus élevée que les femmes. D'après des résultats de cette étude, la dose de dialyse recommandée par séance est de 40 à $45 \mathrm{~L}$ pour les femmes et 45 à $50 \mathrm{~L}$ pour les hommes. Il n'apparaîssait pas évident que des doses plus élevées permettaient une meilleure survie (15). Cependant, les résultats d'une étude observationnelle, prospective, multicentrique, menée chez 6129 patients espagnols ont montré que la prescription de 3 et $9 \mathrm{~L}$ de dose de dialyse supplémentaire à la dose minimale requise réduisait respectivement les risques relatifs de mortalité et d'hospitalisation (16).

Face à cette discordance de pertinence pronostique entre Kt et $\mathrm{V}$, il a été proposé de normaliser le Kt non pas au V mais à la surface corporelle indépendante du sexe (17). Ces études suggèrent la nécessité à majorer les doses de dialyse chez les femmes et les sujets de faible corpulence. D'autres études seraient nécessaires pour déterminer des cibles minimales de $\mathrm{Kt} / \mathrm{SC}$ à atteindre. Il s'avère que les cibles de Kt/V définies actuellement s'adaptent à la majorité de la population et qu'il s'agit de l'indice le plus simple à mesurer. 
A l'issue de ces nombreux travaux, la dose de dialyse cible recommandée par séance est un $\mathrm{Kt} / \mathrm{V}$ équilibré d'au moins 1,2 soit un Kt/V simple pool d'au moins 1,4 (18). De façon à s'assurer que la dose dialytique minimale est délivrée en cas de faible $\mathrm{V}$ malgré un $\mathrm{Kt} / \mathrm{V}$ equlibré supérieur à 1,2, on peut y associer une cible de Kt d'au moins $40 \mathrm{~L}$ chez la femme et $45 \mathrm{~L}$ chez l'homme.

Le Kt/V urée simple pool peut être mesuré de manière indirecte en dosant les taux d'urée plasmatique pré- et postdialytique. Des mesures correctives visant à l'exactitude de la mesure sont ensuite apportées et intégrées dans un modèle mathématique complexe qui requiert des outils informatiques pour le calcul du Kt/V (19). Il est recommandé de mesurer le Kt/V au moins une fois par mois dans une démarche d'assurance qualité.

Au cours des années 2000, des mesures de conductivité en ligne ont été réalisées par des modules embarqués dans les générateurs d'hémodialyse comme substitut de la mesure de la concentration du sodium du dialysat. La dialysance ionique mesurée a été corrélée à la clairance effective de l'urée (K) (20). On peut ainsi mesurer le Kt et le Kt/Vsp à condition de connaître le V du patient (21). Le grand avantage de ces mesures automatisées est de pouvoir être effectué en temps réel, à chaque séance et sans surcoût matériel ni humain. Dans le registre REIN, seulement $61 \%$ des dossiers ont été renseignés pour la variable $\mathrm{Kt} / \mathrm{V} ; 34 \%$ des $\mathrm{Kt} / \mathrm{V}$ renseignés ont été estimés à partir de la dialysance ionique (données issues du registre REIN 2016).

Bien entendu, l'écueil principal de l'utilisation de ces indicateurs comme marqueurs de dialyse adéquate est qu'ils se réfèrent à l'épuration d'une seule molécule de petit poids moléculaire, l'urée. Il est évident que ce paramètre seul n'est pas suffisant pour garantir une dialyse optimale et qu'il faut s'assurer par ailleurs de l'équilibre électrolytique, hydrosodé, nutritionnel, phosphocalcique, hématologique, de l'équilibre tensionnel et de la tolérance hémodynamique des séances de dialyse ainsi que de l'épuration des moyennes molécules. D'autre part, la fonction rénale résiduelle est ignorée par la NCDS et par lesrecommandations de la Kidney Disease Outcomes Quality Initiative (KDOQI). Or, la Netherlands Cooperative Study on the Adequacy of Dialysis (NECOSAD), étude prospective, multicentrique, observationnelle a bien montré la valeur pronostique de la fonction rénale résiduelle sur la survie des patients en hémodialyse chronique (22). Comme l'ont suggéré Charra et al., la survie du patient devrait être considérée comme le meilleur indicateur de dialyse adéquate (23).

Le pourcentage de réduction moyen de l'urée, défini comme la baisse perdialytique des taux d'urée plasmatique divisée par l'urée plasmatique prédialytique a été utilisé comme mesure simplifiée de la dose de dialyse (24). Bien que corrélé au Kt/V , cet index ne reflète pas parfaitement la dose de dialyse et donc l'efficacité du système d'épuration car il n'intègre pas l'ultrafiltration réalisée et dépend en revanche du taux de génération de l'urée. Pour ces raisons, le Kt/V est préféré au pourcentage de réduction moyen de l'urée comme index de quantification de la dose de dialyse délivrée (8). 
Les cibles définies ci-dessus sont valables pour des séances de dialyse conventionnelles de $4 \mathrm{~h}$ trois fois par semaine. L'hémodialyse est caractérisée par un fonctionnement discontinu avec chute rapide de la concentration sanguine de l'urée au cours de la séance de dialyse, suivie d'une remontée progressive jusqu'à la dialyse suivante. A l'état stable, la concentration plasmatique d'urée résulte de l'équilibre entre l'épuration et la génération d'urée. Pour s'affranchir de ces fluctuations, Lowrie et al. ont proposé d'utiliser la concentration plasmatique moyenne hebdomadaire de l'urée (time-averaged concentration, TAC) (6). Ce paramètre ne suffit pas à rendre compte de la dose de dialyse car il dépend de nombreux paramètres : fonction rénale résiduelle, apports protidiques, durée et fréquence des dialyses. La déviation moyenne des concentrations d'urée (time-averaged deviation, TAD) autour de la concentration plasmatique moyenne hebdomadaire de l'urée permet de quantifier l'amplitude des variations des concentrations d'urée, témoin du déséquilibre intérieur (25). De façon à définir une mesure universelle de la dose de dialyse délivrée en fonction de la fréquence hebdomadaire des sessions, Casino et Lopez, puis Gotch ont créé de nouveaux indices : l'equivalent renal urea clearance (EKRc) et le Kt/V standard (std Kt/V) (26,27). L'efficacité de la dialyse est d'autant plus élevée que la fréquence hebdomadaire des séances augmente. En l'absence de résultats précis, les European Best Practice Guidelines ont émis des cibles de dialyse adéquates à atteindre en fonction de la fréquence hebdomadaire des séances de dialyse en tenant compte de la fonction rénale résiduelle : la cible de $\mathrm{Kt} / \mathrm{V}$ standard hebdomadaire à atteindre en hémodialyse quotidienne 6 fois $2 \mathrm{~h}$ par semaine est de 2,8 avec un EKRc de 13,8 mL/min (18).

\section{Prescription de la dose de dialyse diffusive}

La prescription de la dose de dialyse est définie pour des séances conventionnelles de $4 \mathrm{~h}$ trois fois par semaine et n'est pas adaptée aux séances de dialyse de fréquence différente incluant la dialyse incrémentale.

Dans le registre REIN, 22\% des patients ne recevaient pas la dose de dialyse cible puisque le $\mathrm{Kt} / \mathrm{V}$ équilibré était inférieur à 1,2 (données issues du registre REIN 2016).

\subsection{Clairance effective de l'urée $\mathrm{K}$}

Elle représente le volume de sang du patient totalement épuré d'urée par unité de temps $(\mathrm{mL} / \mathrm{min})$. Elle dépend du dialyseur utilisé et de ses conditions d'utilisation.

\subsubsection{Dialyseur utilisé}

Une membrane de dialyse est définie selon plusieurs critères. Le KoA de l'urée correspond au coefficient de transfert de masse du dialyseur pour l'urée par unité de surface. Il dépend principalement des propriétés physiques de chaque dialyseur (porosité, propriétés hydrophiles ou hydrophobes) et est en général communiqué par les fabricants. Pour une membrane donnée, le KoA est d'autant plus élevé que la surface est importante. Cette surface d'échange des solutés est optimisée 
par certains industriels en favorisant la circulation hydraulique du dialysat au sein du dialyseur : mise en place de space filaments, ondulation des fibres, entrée latérale du dialysat sur le dialyseur.

En fonction de la géométrie des dialyseurs, le risque de coagulation des fibres est plus ou moins important, notamment pour les fibres externes. Il est important d'adapter au mieux l'anticoagulation du circuit de manière à garantir la meilleure qualité de restitution du sang. Plus le nombre de fibres du dialyseur coagulent pendant la dialyse et plus la baisse de clairance sera importante (baisse moyenne physiologique de $5 \%$ sur $4 \mathrm{~h}$ liée à l'ultrafiltration et au colmatage des fibres), ce qui génèrera une dose de dialyse Kt plus basse (28).

L'utilisation d'un dialyseur dont la configuration permet la réalisation d'hémodiafiltration interne n'a pas d'influence sur la dose de dialyse. Cependant, son intérêt reste majeur pour l'épuration des solutés de plus haut poids moléculaire que l'urée (29).

\subsubsection{Conditions d'utilisation du dialyseur}

\subsubsection{Débit de pompe à sang $\left(\mathrm{Q}_{\mathrm{b}}\right)$}

L'étude DOPPS a révélé l'obtention de doses de dialyse différentes entre les différents pays. L'obtention de Kt/V cible était plus faible dans les pays du Golfe et au Japon qu'en Europe et en Amérique du Nord. Les pratiques médicales sont différentes entre ces différents pays. Parmi ces différences, il y a la prescription des débits de pompe à sang : plus faibles, en moyenne de 300 $\mathrm{mL} / \mathrm{min}$ dans les pays du golfe et de $195 \mathrm{~mL} / \mathrm{min}$ au Japon contre $326 \mathrm{~mL} / \mathrm{min}$ et $400 \mathrm{~mL} / \mathrm{min}$ en Europe et en Amérique du Nord (30).

Récemment, une étude prospective australienne a montré que l'augmentation des débits de pompe à sang de 252 à $349 \mathrm{~mL} / \mathrm{min}$ permettait l'augmentation significative et linéaire du pourcentage de réduction moyen de l'urée (31).

L'obtention de débits de pompe à sang élevés impose un accès vasculaire de qualité en privilégiant bien sûr la fistule artérioveineuse native. Il est important de surveiller au quotidien tous les indicateurs permettant de dépister une sténose qui justifie un traitement angioplastique ou chirurgical. En effet, la baisse brutale et inexpliquée de la clairance est en lien avec une sténose hémodynamique de la fistule artérioveineuse dans la majorité des cas.

\subsubsection{Débit de dialysat}

Dans les années 1960, le débit dialysat optimal compte tenu de la conformation des dialyseurs utilisés était de $500 \mathrm{~mL} / \mathrm{min}$. Quelques années plus tard, il a été montré que l'augmentation du débit dialysat de 500 à $800 \mathrm{~mL} / \mathrm{min}$ pour un débit de pompe à sang constant à $450 \mathrm{~mL} / \mathrm{min}$ et un même dialyseur permettait d'augmenter de 14\% la dose de dialyse (32). L'influence du débit dialysat sur 
l'épuration des petits solutés est d'autant plus importante que la perméabilité hydraulique de la membrane est élevée (33).

L'adaptation du débit dialysat peut se faire automatiquement à partir du débit de pompe à sang en fonction de la membrane utilisée (AutoFlow [AF] des générateurs 5008 Fresenius Medical Care, Bad Homburg, Germany) dans le but d'optimiser l'épuration tout en minimisant la consommation de dialysat et donc le coût. Une étude récente a comparé la dose de dialyse obtenue chez 33 patients hémodialysés chroniques en fonction de trois débits dialysats, les autres prescriptions de dialyse restant identiques: $500 \mathrm{~mL} / \mathrm{min}, 700 \mathrm{~mL} / \mathrm{min}$ et $\mathrm{AF}$ entre 1,3 et 1,4 soit un débit de dialysat moyen de $404 \pm 29 \mathrm{~mL} / \mathrm{min}, \mathrm{Le} \mathrm{Kt} / \mathrm{V}$ obtenu était respectivement de 1,50 $\pm 0,16,1,52 \pm 0,16$ et 1,49 $\pm 0,15$, Ces résultats suggèrent l'absence d'intérêt majeur à augmenter le débit dialysat au-delà de $500 \mathrm{~mL} / \mathrm{min}$ pour l'obtention de la dose de dialyse cible ce qui permet une économie significative du dialysat utilisé $(34,35)$.

\subsubsection{Connexion $d u$ sang et du dialysat au dialyseur}

Elle doit se faire à contre courant. Cela permet d'optimiser les gradients de concentration d'urée entre le sang et le dialysat tout le long de la membrane et donc d'optimiser la clairance du dialyseur et ainsi la clairance effective (28).

\subsection{Durée (t) de séance de dialyse}

Les résultats de la NCDS ont montré une tendance à l'amélioration pronostique des patients avec l'augmentation de la durée de dialyse $(t)$. Cependant, en l'absence de lien statistiquement significatif $(\mathrm{p}=0,06)$, la communauté néphrologique a longtemps sous estimé ce résultat et de fait a négligé l'importance de la durée de dialyse (6).

La durée de dialyse est bien sûr un déterminant de la dose de dialyse : dans la NCDS, le Kt/Vsp moyen des patients ayant une durée de dialyse courte (temps moyen : 3,2 h) était plus faible que celui des patients ayant une durée moyenne de dialyse de 4,5 heures $=0,75$ versus 0,90 (7). Plus tard, les résultats de la DOPPS ont confirmé l'augmentation concomitante du Kt/V avec l'augmentation de la durée de dialyse quelque soit la région étudiée (Japon, Europe, Etats Unis).

D'autre part, les études réalisées à partir des registres ANZDATA ou DOPPS ont montré que des durées de dialyse longues (de plus de 240 minutes), réalisées chez des patients hémodialysés chroniques trois fois par semaine étaient significativement associées à une réduction de mortalité indépendamment de la dose de dialyse délivrée (36,37). Dans la DOPPS, chaque augmentation de 30 minutes de la durée de dialyse permettait de réduire la mortalité de $7 \%$. $(p<0,0001)$. Ce bénéfice à l'amélioration de la survie avec l'allongement des séances était plus important au Japon. 
Ce qu'il faut retenir de ces études est qu'il ne faut pas réduire la durée de dialyse sous prétexte que le Kt/V est obtenu. Or, selon le registre REIN, 18,2\% des patients hémodialysés chroniques trois fois par semaine ont des durées de séance inférieures à 4 h (données issues du registre REIN 2016). En effet, le prolongement de la séance permet d'épurer des molécules de poids moléculaire plus élevé, d'améliorer le contrôle de la phosphatémie et de l'acidose métabolique et surtout d'améliorer la stabilité hémodynamique de la séance avec un meilleur contrôle de l'équilibre hydrosodé, un meilleur contrôle tensionnel, une baisse des taux d' ultrafiltration horaire et donc une réduction des épisodes hypotensifs intradialytiques (36).

\subsection{Volume de distribution de l'urée (V)}

L'estimation en temps réel de la dose de dialyse Kt est aujourd'hui possible sur plusieurs générateurs de dialyse à partir de la mesure de la dialysance ionique. L'estimation à chaque séance du Kt/Vsp est donc possible à condition de renseigner le volume de distribution de l'urée à l'équilibre $(V)$, assimilé à l'eau totale.

Le volume de distribution de l'urée à l'équilibre peut être estimé de plusieurs façons : par mesure anthropométrique en utilisant la formule de Watson $\left(V_{\mathrm{WAT}}\right)$, par mesure impédancemétrique $\left(V_{\mathrm{IMP}}\right)$, par estimation indirecte à partir du calcul du Kt/Vsp selon la formule de Daugirdas de deuxième génération $\left(V_{\mathrm{DAUG}}=\mathrm{Kt}\right.$ séance dialysance ionique $\left./(\mathrm{Kt} / \mathrm{V}) \mathrm{sp}\right)$ et par quantification directe de la dialyse $\left(V_{\mathrm{DDQ}}\right)$, méthode de référence.

Une étude a comparé les valeurs de $V_{\mathrm{WAT}}, V_{\mathrm{IMP}}$ (BCM Fresenius Medical Care) et $V_{\mathrm{DAUG}}$ à $V_{\mathrm{DDQ}}$, La formule de Watson surestimait de $20 \%$ l'eau totale du patient et donc sous-estimait de $20 \%$ le $\mathrm{Kt} / \mathrm{Vsp}$ estimé par les moniteurs de dialyse. Le $V_{\mathrm{IMP}}$ était légèrement inférieur à $V_{\mathrm{DDQ}}$ tandis que le $V_{\mathrm{DAUG}}$ était plus proche mais la corrélation et la concordance au $V_{\mathrm{DDQ}}$ étaient plus fortes avec $V_{\mathrm{IMP}}$ qu'avec $V_{\text {Daug. }}$ Ces deux méthodes peuvent donc être utilisées de façon simple (38).

Le Kt/V ainsi mesuré est corrélé au Kt/V calculé à partir des concentrations plasmatiques d'urée pré- et postdialytique (39).

\section{Prescription de la dose de dialyse convective}

\subsection{Hémodiafiltration : description de la technique}

L'hémodiafiltration est une technique d'épuration extrarénale qui associe à la composante diffusive une composante convective permettant d'optimiser l'épuration des solutés de haut poids moléculaire (de plus de $5 \mathrm{kD}$ ). Depuis la circulaire du 30 janvier 2007, il est devenu possible de pratiquer l'hémodiafiltration en ligne dans les établissements de santé dans des conditions fiables et sécuritaires. Cela a permis l'essor de la technique en France : la prévalence d' hémodiafiltration en ligne était de 32,3\% en 2016 contre 5,4\% en 2006 (données issues du registre REIN de 2016 et 2006). 
La convection repose sur une filtration de l'eau plasmatique régulée par la pompe d'ultrafiltration et la pression transmembranaire appliquée au sang du patient. La fraction filtrée de l'eau plasmatique correspond au rapport du débit d'ultrafiltration sur le débit d'eau plasmatique $\left(\mathrm{Q}_{\mathrm{u}} / \mathrm{Q}_{\mathrm{ep}}\right)$. Cette fraction filtrée dépend de la modalité d' hémodiafiltration. En postdilution, l'hémoconcentration limite la fraction filtrée à $50 \%$ de du débit d'eau plasmatique $\mathrm{Q}_{\mathrm{ep}}$ à cause du risque de coagulation de la membrane. En prédilution, la fraction filtrée est de $100 \%$ du débit d'eau plasmatique.

En pratique clinique, la fraction filtrée est souvent exprimée comme le rapport du $\mathrm{Q}_{\mathrm{uf}} \operatorname{sur} \mathrm{Q}_{\mathrm{b}}$ et dépend donc des conditions rhéologiques sanguines (concentration protides et taux d'hématocrite): elle est de 25 à $30 \%$ du $\mathrm{Q}_{\mathrm{b}}$ en postdilution et de $50 \%$ du $\mathrm{Q}_{\mathrm{b}}$ en prédilution (40). La substitution se fait de façon simultanée à la filtration et est isovolumique au volume filtré à laquelle on soustrait l'ultrafiltration liée à la perte de poids (UF). En postdilution, la substitution se fait en aval du dialyseur juste en amont du piège à bulle veineux. En prédilution, la substitution se fait en amont du dialyseur. Il existe des modalités mixtes: la mixed-dilution et la mid-dilution. L' hémodiafiltration en ligne postdilution est la modalité la plus performante et la seule ayant montré un bénéfice sur la survie des patients.

En hémodiafiltration, la diffusion et la convection sont réalisées simultanément sans être simplement additives car elles interagissent entre elles : la diffusion réduit la concentration des petits solutés et par conséquent la masse épurée par convection et la convection réduit le débit sanguin et ainsi la force permettant la gestion des transferts diffusifs. En hémodiafiltration en ligne, sur certains générateurs, la convection réduit le $\mathrm{Q}_{\mathrm{d}}$ puisqu'une partie de ce liquide est dédié à la substitution. Ainsi, la convection ne permet pas d'optimiser l'épuration des molécules de petit poids moléculaire alors que son importance est majeure dans l'épuration des solutés de moyen ou haut poids moléculaire. Des modèles mathématiques suggèrent que 40 à $50 \%$ du taux d'ultrafiltration réalisé représente la clairance convective additive à la clairance diffusive. La convection est à prioriser en appliquant une fraction filtrée maximale (40).

\subsection{Objectifs d'épuration par hémodiafiltration en ligne}

La cible de dose diffusive est la même qu'en hémodialyse conventionnelle. La dose convective est représentée par le volume convectif : il s'agit de la somme du volume substitué et de l'ultrafiltration liée à la perte de poids. Il est le meilleur représentant de la dose convective.

En 2006, Canaud et al. ont montré à partir de l'étude observationnelle DOPPS que l' hémodiafiltration postdilution permettait de réduire la mortalité de $35 \%$ par rapport à l'hémodialyse conventionnelle à condition d'obtenir des volumes filtrés de plus de 15 litres (41). Quelques années plus tard, trois études prospectives randomisées comparant l' hémodiafiltration en ligne à 
l'hémodialyse, bien qu'ayant des résultats divergents suggéraient toutes que le traitement par hémodiafiltration postdilution était associé à une amélioration de la survie, à la condition d'obtenir des volumes convectifs élevés (cible plus de 23,1 1 dans Estudio de Supervivencia de Hemodiafiltración Online [ESHOL]) (42-44). Les résultats d'une étude consolidée reprenant ces trois études et la French National Study ont confirmé à partir des données de 2793 patients suivis pendant 2,5 ans un bénéfice de l' hémodiafiltration en ligne postdilution sur la survie des patients, avec une réduction de $14 \%$ de la mortalité de toute cause et de $22 \%$ si le volume convectif indexé à la surface corporelle était supérieur à 231/1,73 $\mathrm{m}^{2}$ (42). On peut donc à ce jour considérer que la cible de volume convectif à atteindre en hémodiafiltration en ligne postdilution doit être d'au moins 23,1 1 tout en étant supérieure à 231 pour $1,73 \mathrm{~m}^{2}$ de surface corporelle.

\subsection{Déterminants du volume convectif}

Penne et al. ont étudié chez 235 patients participant à l'étude CONTRAST les déterminants du volume convectif (46). En analyse multivariée, les facteurs associés à un volume convectif élevé étaient un débit de pompe à sang élevé, la durée de la séance et les conditions rhéologiques.

\subsubsection{Un débit de pompe à sang élevé}

Seuls les patients ayant un $\mathrm{Q}_{\mathrm{b}}$ supérieur à $370 \mathrm{~mL} / \mathrm{min}$ atteignent des volumes convectifs d'au moins 201.

Les volumes convectifs moyens étaient de 23,7 1 dans ESHOL et de 20,7 1 dans les études CONTRAST et TURKISH. Ces différences étaient essentiellement liées aux $\mathrm{Q}_{\mathrm{b}}: 387 \mathrm{~mL} / \mathrm{min}$ dans ESHOL, 300 et $310 \mathrm{~mL} / \mathrm{min}$ respectivement dans CONTRAST et TURKISH.

Pour obtenir des $\mathrm{Q}_{\mathrm{b}}$ élevés, il faut utiliser un bon abord vasculaire. L' abord vasculaire de choix est la fistule artérioveineuse mais il est tout à fait possible de faire de l' hémodiafiltration en ligne avec des volumes convectifs élevés sur un cathéter. Dans l'ESHOL, 7,5\% des patients en hémodiafiltration en ligne étaient dialysés sur cathéter.

\subsubsection{Durée de la séance}

Dix minutes de séance supplémentaires permettent de majorer le volume convectif de 0,9 1 .

\subsubsection{Conditions rhéologiques}

Il existe une corrélation positive du volume convectif avec l'albuminémie (optimisation du remplissage plasmatique) et négative avec l'hématocrite (diminution de la disponibilité de l'eau plasmatique). 


\subsection{Dialyseur en hémodiafiltration en ligne}

En hémodiafiltration en ligne, les membranes synthétiques utilisées doivent être de grande surface, de haute perméabilité, avec coefficient de tamisage pour la $\beta 2$ microglobuline élevé, sans fuite d'albumine.

\subsubsection{Surface de la membrane}

Wizemann et al. ont montré qu'une membrane de 2,2 $\mathrm{m}^{2}$ permettait d'augmenter de $22 \%$ la clairance de la $\beta 2$ microglobuline en comparaison à une même membrane de surface $1,4 \mathrm{~m}^{2}$ (47).

\subsubsection{Perméabilité}

Le caractère hautement perméable d'une membrane se définit par un $\mathrm{K}_{\mathrm{UF}}$ supérieur ou égal à 20 $\mathrm{ml} / \mathrm{h} / \mathrm{mmHg}$. Cette perméabilité correspond à la perméabilité hydraulique mais pas à celle des solutés. Il est possible d'obtenir de hauts volumes convectifs grâce à une membrane de perméabilité hydraulique élevée sans épurer correctement les moyennes molécules. La $\beta 2$ microglobuline est le représentant de ces dernières. Dans Hemo Study, il a été montré une élévation de $11 \%$ de la mortalité pour une augmentation de $10 \mathrm{mg} / \mathrm{l}$ de la $\beta 2$ microglobuline au delà du seuil de $27,5 \mathrm{mg} / \mathrm{l}$. C'est cette cible qui est à atteindre (48).

\subsubsection{Coefficient de tamisage pour la $\beta 2$ microglobuline}

Ouseph et al. ont montré que deux membranes high flux en polysulfone d'1,7 m² n'étaient pas équivalentes en termes d'épuration des solutés de poids moléculaire moyen : le pourcentage de réduction plasmatique de la $\beta 2$ microglobuline était de 39\% avec l'une et de 54\% avec l'autre (49). Le groupe EUDIAL a ainsi défini deux critères d'exigence concernant le choix d'un dialyseur en hémodiafiltration en ligne : en plus d'être de haute perméabilité hydraulique, il doit avoir un coefficient de tamisage de la $\beta 2$ microglobuline supérieur à 0,6 (40).

\subsubsection{Sans fuite d'albumine}

Selon les recommandations japonaises de Kim, un dialyseur en hémodiafiltration en ligne doit permettre une réduction du taux plasmatique de la $\beta 2$ microglobuline de plus de $80 \%$ et de la myoglobine de plus de 65\% sans entrainer plus de $5 \mathrm{~g}$ de perte d'albumine par séance de dialyse (50). Ahrenholz et al. ont montré que des membranes équivalentes en terme d'épuration de la $\beta 2$ microglobuline (70\% de réduction plasmatique) pouvaient perdre entre 1 et $7 \mathrm{~g}$ par séance (51). Il paraît donc essentiel en hémodiafiltration en ligne de tester in vivo les dialyseurs proposés par l'industrie afin d'évaluer leurs performances d'épuration et leur caractère sécuritaire. 


\subsection{Automatisation du débit de substitution}

La substitution peut être réalisée selon deux modalités dépendantes des générateurs, le mode «volume contrôle » et le mode «pression contrôle » (52).

\subsubsection{Mode «volume contrôle »}

Une fraction filtrée et donc un volume substitué fixes sont prescrits pour toute la durée de la séance de dialyse. En conséquence, la pression transmembranaire augmente en réponse à l'hémoconcentration et à la formation progressive du protéine cake à la surface interne de la membrane. Des alarmes intempestives de pression transmembranaire peuvent survenir.

\subsubsection{Mode «pression contrôle »}

La pression transmembranaire est paramétrée entre deux valeurs, minimale (valeur équivalente à la convection initiale obtenue en volume contrôle) et maximale (cible supérieure permettant de maintenir les performances du dialyseur). La fraction filtrée est régulée tout au long de la séance de façon à maintenir la pression transmembranaire entre ces deux valeurs cibles. Du fait d'une filtration accrue en début de séance favorisée par une cible pression transmembranaire maximale élevée, les pertes d'albumine peuvent être importantes.

\subsubsection{Générateurs}

Il en existe deux types :

- L'autosub (Générateur 5008, Fresenius Medical Care, Bad Homburg, Allemagne) : la fraction filtrée est ajustée au $\mathrm{K}_{\mathrm{UF}}$ du dialyseur et à l'hémoconcentration. Cette dernière est estimée à partir du taux de protides renseigné en début de séance, du taux d'hématocrite déterminé en continu par le module Blood Volume Monitor (BVM) et du taux d' ultrafiltration lié à la perte de poids.

- L'autosub plus (Générateur Cordiax 5008, Fresenius Medical Care, Bad Homburg, Allemagne) : la fraction filtrée est adaptée à l'hémoconcentration déterminée grâce à l'analyse en continu d'un signal d'impulsions de pression généré par la pompe à sang. Ce signal traverse ensuite le dialyseur et est enregistré par le capteur de PV. Son analyse répond ensuite au principe de la «transformée de Fourier ».

- L'ultracontrol (Gambro/Hospal, Lund, Suède) : Le moniteur détermine progressivement au début de chaque séance une pression transmembranaire optimale permettant d'atteindre la fraction filtrée maximale. Ce scan de la pression transmembranaire est réalisé toutes les heures de façon à s'adapter à l'hémoconcentration et aux modifications perdialytiques des performances du dialyseur. 
L'automatisation du débit de substitution permet, premièrement, d'optimiser les volumes substitués tout en diminuant significativement les contraintes liées à l'hémoconcentration et deuxièmement, de diminuer le nombre d'alarmes pendant la séance (53).

\section{Conclusion}

La prescription de la dose de dialyse doit s'inscrire dans une démarche assurance qualité. Les paramètres de prescription sont justifiés par l'obtention de cibles définies $(\mathrm{Kt} / \mathrm{V} \mathrm{sp}>1,4$ et $\mathrm{Kt}>40$ et 451 pour les femmes et les hommes respectivement, volume convectif supérieur à 23,11 et 23 1/1,73 $\mathrm{m}^{2}$ ). Il faut vérifier la conformité des doses diffusives et / ou convectives obtenues et mettre en place des mesures correctives le cas échéant.

\section{Références bibliographiques}

1. Scribner BH, Buri R, Caner JE, Hegstrom R, Burnell JM. The treatment of chronic uremia by means of intermittent hemodialysis: a preliminary report. Trans Am Soc Artif Intern Organs 1960;6:114-22.

2. De Palma JR, Bolton CF, Baltzan MA, Baltzan RB. Adequate hemodialysis schedule. N Engl J Med 1971;285(6):353-4.

3. Babb AL, Popovich RP, Christopher TG, Scribner BH. The genesis of the square meter-hour hypothesis. Trans Am Soc Artif Intern Organs 1971;17:81-91.

4. Twardowski ZJ, Misra M. A need for a paradigm shift in focus: From Kt/Vurea to appropriate removal of sodium (the ignored uremic toxin). Hemodial Int Int Symp Home Hemodial 2018;22(S2):S29-64.

5. Kjellstrand CM, Evans RL, Petersen RJ, Shideman JR, von Hartitzsch B, Buselmeier TJ. The « unphysiology » of dialysis: a major cause of dialysis side effects? Kidney Int Suppl 1975;(2):30-4.

6. Lowrie EG, Laird NM, Parker TF, Sargent JA. Effect of the hemodialysis prescription of patient morbidity: report from the National Cooperative Dialysis Study. N Engl J Med 1981;305(20):1176-81.

7. Gotch FA, Sargent JA. A mechanistic analysis of the National Cooperative Dialysis Study (NCDS). Kidney Int 1985;28(3):526-34.

8. NKF-DOQI clinical practice guidelines for hemodialysis adequacy. National Kidney Foundation. Am J Kidney Dis 1997;30(3 Suppl 2):S15-66.

9. Daugirdas JT, Depner TA, Gotch FA, Greene T, Keshaviah P, Levin NW, et al. Comparison of methods to predict equilibrated Kt/V in the HEMO Pilot Study. Kidney Int 1997;52(5):1395-405. 
10. Eknoyan G, Beck GJ, Cheung AK, Daugirdas JT, Greene T, Kusek JW, et al. Effect of dialysis dose and membrane flux in maintenance hemodialysis. N Engl J Med 2002;347(25):2010-9.

11. Saran R, Canaud BJ, Depner TA, Keen ML, McCullough KP, Marshall MR, et al. Dose of dialysis: key lessons from major observational studies and clinical trials. Am J Kidney Dis 2004;44(5 Suppl 2):47-53.

12. Kopple JD, Zhu X, Lew NL, Lowrie EG. Body weight-for-height relationships predict mortality in maintenance hemodialysis patients. Kidney Int 1999;56(3):1136-48.

13. Chertow GM, Lazarus JM, Lew NL, Ma L, Lowrie EG. Development of a population-specific regression equation to estimate total body water in hemodialysis patients. Kidney Int 1997;51(5):1578-82.

14. Price DA, Owen WF. African-Americans on maintenance dialysis: a review of racial differences in incidence, treatment, and survival. Adv Ren Replace Ther 1997;4(1):3-12.

15. Lowrie EG, Chertow GM, Lew NL, Lazarus JM, Owen WF. The urea [clearance $\times$ dialysis time] product (Kt) as an outcome-based measure of hemodialysis dose. Kidney Int 1999;56(2):729-37.

16. Maduell F, Ramos R, Varas J, Martin-Malo A, Molina M, Pérez-Garcia R, et al. Hemodialysis patients receiving a greater $\mathrm{Kt}$ dose than recommended have reduced mortality and hospitalization risk. Kidney Int 2016;90(6):1332-41.

17. Lowrie EG, Li Z, Ofsthun N, Lazarus JM. The online measurement of hemodialysis dose (Kt): clinical outcome as a function of body surface area. Kidney Int 2005;68(3):1344-54.

18. Tattersall J, Martin-Malo A, Pedrini L, Basci A, Canaud B, Fouque D, et al. EBPG guideline on dialysis strategies. Nephrol Dial Transplant2007;22 Suppl 2:ii5-21.

19. Gotch FA. Urea kinetic modelling. Nephrol Dial Transplant1995;10(12):2378-9.

20. Lindsay RM, Bene B, Goux N, Heidenheim AP, Landgren C, Sternby J. Relationship between effective ionic dialysance and in vivo urea clearance during hemodialysis. Am J Kidney Dis sept 2001;38(3):565-74.

21. Petitclerc T, Coevoet B. Dialysance ionique et qualité contrôle de qualité de l'épuration en hémodialyse. Nephrologie 2001;22(5):191-7.

22. Termorshuizen F, Dekker FW, van Manen JG, Korevaar JC, Boeschoten EW, Krediet RT, et al. Relative contribution of residual renal function and different measures of adequacy to survival in 
hemodialysis patients: an analysis of the Netherlands Cooperative Study on the Adequacy of Dialysis (NECOSAD)-2. J Am Soc Nephrol 2004;15(4):1061-70.

23. Charra B, Calemard E, Ruffet M, Chazot C, Terrat JC, Vanel T, et al. Survival as an index of adequacy of dialysis. Kidney Int 1992;41(5):1286-91.

24. Basile C, Casino F, Lopez T. Percent reduction in blood urea concentration during dialysis estimates Kt/V in a simple and accurate way. Am J Kidney Dis 1990;15(1):40-5.

25. Lopot F, Válek A. Time-averaged concentration--time-averaged deviation: a new concept in mathematical assessment of dialysis adequacy. Nephrol Dial Transplant 1988;3(6):846-8.

26. Casino FG, Lopez T. The equivalent renal urea clearance: a new parameter to assess dialysis dose. Nephrol Dial Transplant 1996;11(8):1574-81.

27. Gotch FA. The current place of urea kinetic modelling with respect to different dialysis modalities. Nephrol Dial Transplant 1998;13 Suppl 6:10-4.

28. Tangvoraphonkchai K, Davenport A. Enhancing dialyser clearance-from target to development. Pediatr Nephrol 2017;32(12):2225-33.

29. Locatelli F, Manzoni C, Di Filippo S. The importance of convective transport. Kidney Int Suppl 2002;(80):115-20.

30. AlYousef A, AlGhareeb S, Al Wakeel J, Al-Ghamdi SMG, Bieber BA, Hassan M, et al. Hemodialysis delivery, dialysis dose achievement, and vascular access types in hemodialysis patients from the Gulf Cooperation Council countries enrolled in the dialysis outcomes and practice patterns study phase 5 (2012-2015). Saudi J Kidney Dis Transplant 2016;27(6 Suppl 1):S42-50.

31. Ryan P, Le Mesurier L, Adams K, Choi P, Chacko B. Effect of increased blood flow rate on hemodialysis tolerability and achieved urea reduction ratio. Ther Apher Dial 2018;22(5):494-502.

32. Leypoldt JK, Cheung AK, Agodoa LY, Daugirdas JT, Greene T, Keshaviah PR. Hemodialyzer mass transfer-area coefficients for urea increase at high dialysate flow rates. The Hemodialysis (HEMO) Study. Kidney Int 1997;51(6):2013-7.

33. Leypoldt JK, Cheung AK. Increases in mass transfer-area coefficients and urea Kt/V with increasing dialysate flow rate are greater for high-flux dialyzers. Am J Kidney Dis 2001;38(3):575-9. 34. Alayoud A, Benyahia M, Montassir D, Hamzi A, Zajjari Y, Bahadi A, et al. A model to predict optimal dialysate flow. Ther Apher Dial 2012;16(2):152-8. 
35. Albalate M, Pérez-García R, de Sequera P, Corchete E, Alcazar R, Ortega M, et al. Is it useful to increase dialysate flow rate to improve the delivered Kt? BMC Nephrol 2015;16:20.

36. Marshall MR, Byrne BG, Kerr PG, McDonald SP. Associations of hemodialysis dose and session length with mortality risk in Australian and New Zealand patients. Kidney Int 2006;69(7):1229-36.

37. Saran R, Bragg-Gresham JL, Levin NW, Twardowski ZJ, Wizemann V, Saito A, et al. Longer treatment time and slower ultrafiltration in hemodialysis: associations with reduced mortality in the DOPPS. Kidney Int 2006;69(7):1222-8.

38. Koubaa A, Potier J, de Préneuf H, Queffelou G, Garcia F, Petitclerc T. Estimation du volume de distribution de l'urée chez le patient hémodialysé. Nephrol Ther 2010;6(6):532-6.

39. Créput C, Toledano D, Petitclerc T. Ionic dialysance and determination of $\mathrm{Kt} / \mathrm{V}$ in on-line hemodiafiltration with simultaneousiseppre- and post-dilution. Int J Artif Organs. 2013;36(5):327-34.

40. Tattersall JE, Ward RA, EUDIAL group. Online haemodiafiltration: definition, dose quantification and safety revisited. Nephrol Dial Transplant 2013;28(3):542-50.

41. Canaud B, Bragg-Gresham JL, Marshall MR, Desmeules S, Gillespie BW, Depner T, et al. Mortality risk for patients receiving hemodiafiltration versus hemodialysis: European results from the DOPPS. Kidney Int 2006;69(11):2087-93.

42. Ok E, Asci G, Toz H, Ok ES, Kircelli F, Yilmaz M, et al. Mortality and cardiovascular events in online haemodiafiltration (OL-HDF) compared with high-flux dialysis: results from the Turkish OLHDF Study. Nephrol Dial Transplant 2013;28(1):192-202.

43. Grooteman MPC, van den Dorpel MA, Bots ML, Penne EL, van der Weerd NC, Mazairac AHA, et al. Effect of online hemodiafiltration on all-cause mortality and cardiovascular outcomes. J Am Soc Nephrol 2012;23(6):1087-96.

44. Maduell F, Moreso F, Pons M, Ramos R, Mora-Macià J, Carreras J, et al. High-efficiency postdilution online hemodiafiltration reduces all-cause mortality in hemodialysis patients. J Am Soc Nephrol 2013;24(3):487-97.

45. Peters SAE, Bots ML, Canaud B, Davenport A, Grooteman MPC, Kircelli F, et al. Haemodiafiltration and mortality in end-stage kidney disease patients: a pooled individual participant data analysis from four randomized controlled trials. Nephrol Dial Transplant 2016;31(6):978-84.

46. Penne EL, van der Weerd NC, Bots ML, van den Dorpel MA, Grooteman MPC, Lévesque R, et al. Patient- and treatment-related determinants of convective volume in post-dilution haemodiafiltration in clinical practice. Nephrol Dial Transplant 2009;24(11):3493-9. 
47. Wizemann V, Külz M, Techert F, Nederlof B. Efficacy of haemodiafiltration. Nephrol Dial Transplant 2001;16 Suppl 4:27-30.

48. Cheung AK, Rocco MV, Yan G, Leypoldt JK, Levin NW, Greene T, et al. Serum beta-2 microglobulin levels predict mortality in dialysis patients: results of the HEMO study. J Am Soc Nephrol 2006;17(2):546-55.

49. Ouseph R, Hutchison CA, Ward RA. Differences in solute removal by two high-flux membranes of nominally similar synthetic polymers. Nephrol Dial Transplant 2008;23(5):1704-12.

50. Kim ST. Characteristics of protein removal in hemodiafiltration. Contrib Nephrol 1994;108:23-37. 51. Ahrenholz PG, Winkler RE, Michelsen A, Lang DA, Bowry SK. Dialysis membrane-dependent removal of middle molecules during hemodiafiltration: the beta2-microglobulin/albumin relationship. Clin Nephrol 2004;62(1):21-8.

52. Teatini U, Steckiph D, Romei Longhena G. Evaluation of a new online hemodiafiltration mode with automated pressure control of convection. Blood Purif. 2011;31(4):259-67.

53. Maduell F, Arias M, Garro J, Vera M, Fontseré M, Barros X, et al. [Guidelines for automated manual infusion: a practical way of prescribing postdilution on-line hemodiafiltration]. Nefrol 2010;30(3):349-53. 